\title{
Interaction of ICMEs with the Solar Wind
}

\author{
Pascal Démoulin \\ Observatoire de Paris, LESIA, UMR 8109 (CNRS), F-92195 Meudon Principal Cedex, France
}

\begin{abstract}
Interplanetary Coronal Mass Ejections (ICMEs) are formed of plasma and magnetic field launched from the Sun into the Solar Wind (SW). These coherent magnetic structures, frequently formed by a flux rope, interact strongly with the SW. Such interaction is reviewed by comparing the results obtained from in situ observations and with numerical simulations. Like fast ships in the ocean, fast ICMEs drive an extended shock in front. However, their interaction with the SW is much more complex than that of the ship analogy. For example, as they expand in all directions while traveling away from the Sun, a sheath of SW plasma and magnetic field accumulates in front, which partially reconnects with the ICME magnetic field. Furthermore, not only do ICMEs have a profound impact on the heliosphere, but the type of SW encountered by an ICME has an important impact on its evolution (e.g. increase of mass, global deceleration, lost of magnetic flux and helicity, distortion of the configuration).
\end{abstract}

Keywords: Coronal Mass Ejections, Magnetic cloud, Magnetic field, Solar Wind

PACS: $96.50 . \mathrm{Uv}, 96.60 . \mathrm{ph}, 96.60 . \mathrm{Vg}$

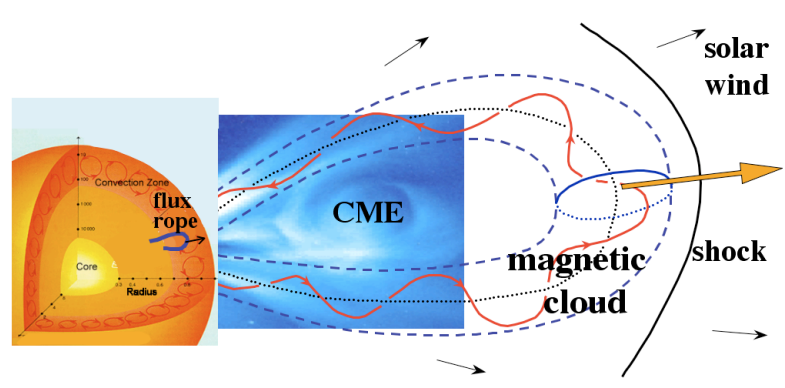

FIGURE 1. Schema showing three time steps. First, the crossing of the convective zone by a flux rope. Second, the launch of a CME (the photospheric emergence and the coronal evolution before the CME are not shown). Finally, depending on the speed and launch direction, the CME can be detected a few days later in the interplanetary space as a magnetic cloud (with a flux rope topology, as shown schematically), or more generally as an ICME. The CME image is from SOHO/LASCO.

\section{INTRODUCTION}

The evolution of an ICME in the SW is part of a long series of physical processes (figure 1). It starts with the amplification of the magnetic field deep in the convective zone and its storage just underneath. At some point, the magnetic field becomes buoyantly unstable, a twisted flux rope is formed, crosses the convective zone and then emerges at the photospheric level. This field is then processed and stored for a few days to a few weeks in the corona, before getting unstable and forming a new flux rope which is ejected from the Sun as a CME. Then, this magnetized plasma, called ICME, travels within and interacts with the SW.

ICMEs are defined by one or several criteria [e.g. 1,2],

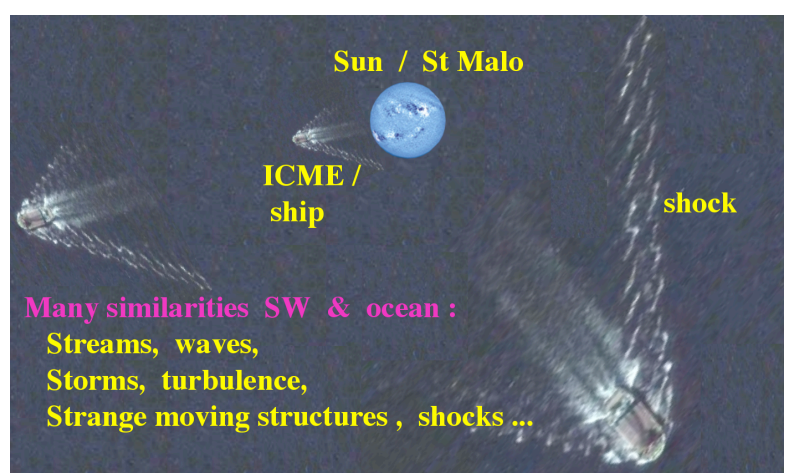

FIGURE 2. Analogies between CMEs / fast ships traveling in the SW / ocean. Compare the shock extension / shape to the one found in MHD simulations (e.g. figures 8a, 9 and 11a).

see also [3, 4, for reviews]. Typical criteria are: (1) a proton temperature at least lower by a factor 2 than in ambient SW with the same velocity; (2) an enhanced helium abundance $(\mathrm{He} / \mathrm{H} \geq 6 \%)$; (3) the presence of counterstreaming suprathermal $(>80 \mathrm{eV})$ electron beams; (4) enhanced ion charge states; (5) a stronger magnetic field with lower variance than in the surrounding SW; (6) a low proton plasma $\beta_{p}(<0.1)$; (7) a smooth and large rotation of the magnetic field. Magnetic clouds (MCs) are a sub-class of ICMEs with criteria 1, 5, 6, and 7 satisfied [5]. Their magnetic configuration, a flux rope, and their physical properties are typically better understood (but still partially!) than for the broader class of ICMEs.

Despite the difference of medium, there are many physical analogies between an ocean and the SW (figure 2). So, the location of the conference, St Malo, was an idea place to study the SW! In particular, fast mov- 


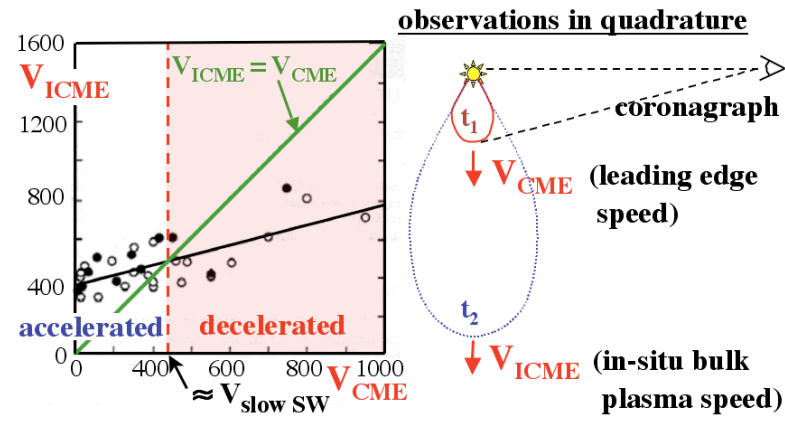

FIGURE 3. Comparison of the velocity of CMEs and their associated ICMEs as realized with coronagraph and in situ observations in quadrature [Adapted from 9].

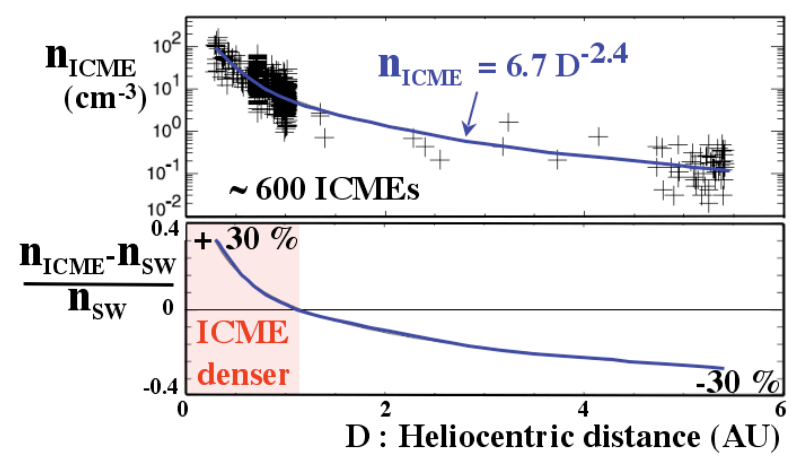

FIGURE 4. Evolution of the density of ICMEs versus the distance to the Sun. The lower panel shows the average evolution of the ICME density relative to the ambient SW density [Adapted from 10].

ing structures are present in both media. They drive forward shocks which extend on a much larger scale than the moving object itself (see e.g. the MHD simulations of $[6,7,8])$.

A main difference between ships and ICMEs is that ICMEs are strongly affected by their interaction with the surrounding medium, in particular their velocity (section 2), their expansion rate (section 3) and their shape (section 4). Like in the ocean, where corsairs could overtake ships, some ICMEs are overtaken by a fast SW stream or by another ICME (section 5). Finally, the interaction of an ICME with the SW changes its main global physical quantities (section 6).

\section{MODIFICATION OF THE ICME MEAN VELOCITY}

One possibility to track an ICME is to follow its leading shock through its radio emission at the local plasma frequency or its harmonic $(\propto \sqrt{\text { density }}$, interplanetary type II emissions). Since the SW plasma density decrease
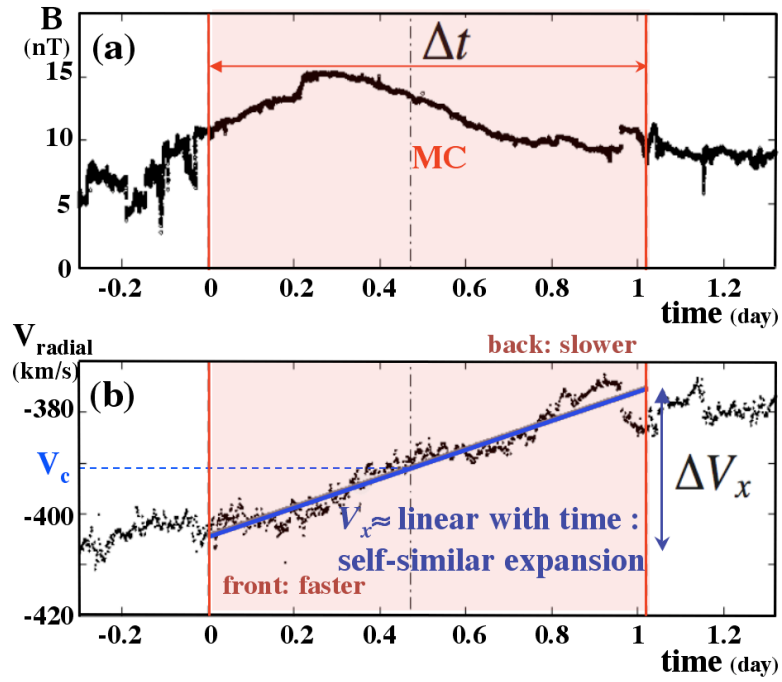

FIGURE 5. (a) Field strength and (b) radial velocity measured at $1 \mathrm{AU}$ in a typical magnetic cloud (MC). The definition of the main quantities defining the undimensioned expansion parameter $\zeta$ are shown (see section 3). [Adapted from 22].

as $\approx 1 / D^{2}$, where $D$ is the solar distance, the inverse of the radio frequency emitted is an estimation of $D$. Then, the shock propagation is followed by the drift in frequency $[11,12,13]$.

Another way to track an ICME is to observe the associated CME with a coronagraph at the solar limb and the in situ ICME with a spacecraft in quadrature with the coronagraph (figure 3 ). This configuration was realized in several special cases in the past [e.g. Solwind, SMM and Helios spacecraft, 9], and more systematically presently with the STEREO spacecraft [14]. ICMEs that are faster (slower) than the surrounding SW are typically decelerated (accelerated), implying a strong coupling (drag force) between the moving structure and the SW $[9,15,16,17]$. These results are coherent with those found with Doppler scintillation measurements using at least two spacecraft [18].

Various drag forces have been investigated [19, 20]. Typically the strongest deceleration occurs close to the Sun, and ICMEs have a nearly constant velocity in most of the heliosphere [outward of $0.3 \mathrm{AU}, 1,2,10$ ]. A fraction of the drag force is due to the accumulation of the slower SW mass in front of the CME. As an extreme example, in an MHD simulation of a fast CME [21], the sum of the CME and piled up mass increases by a factor 5 , inducing a decrease of a factor 3 of its mean velocity (from 1200 to $\approx 400 \mathrm{~km} / \mathrm{s}$ ). 


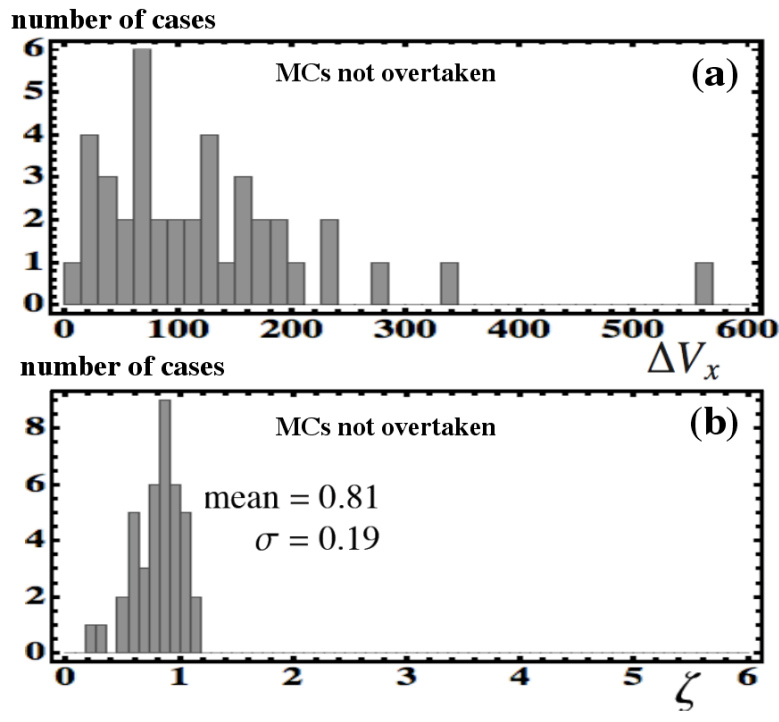

FIGURE 6. (a) Histograms of $\Delta V_{x}$ and (b) $\zeta$ for unperturbated MCs (not overtaken by a fast stream or an ICME). In this sample, all MCs are expanding. [Made from data in 22].
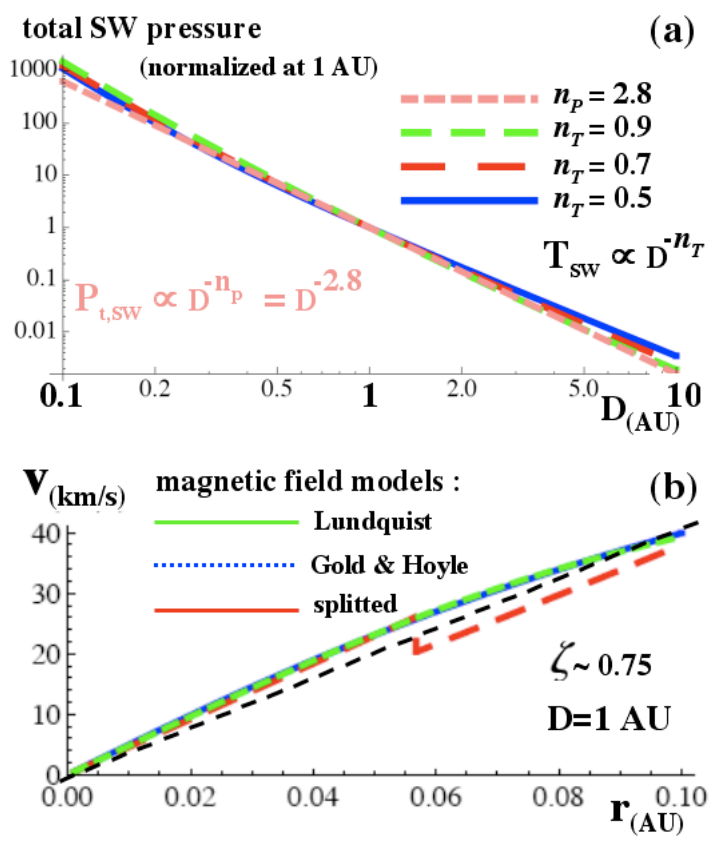

FIGURE 7. (a) Typical evolution of the total pressure, $P_{\mathrm{t}, \mathrm{SW}}$, present in the SW with radial distance to the Sun $(D)$. The plasma pressure is computed with a temperature dependance taken as $D^{-n_{T}}$. The value of $n_{T}$, selected within the observed range, has a small effect on the total pressure which can be approximated with a power law $\left(D^{-n_{P}}\right)$ (b) Results of forcefree flux rope models studying the evolution within a SW with a total pressure $\propto D^{-2.8}$. Three magnetic field profiles across the flux rope are shown. The expansion velocity, $V$, is nearly a linear function of the internal radius, $r$, independently of the magnetic field distribution, as observed in MCs (e.g. figure 5b) [Adapted from 23].

\section{EXPANSION RATE OF ICMES}

The plasma density in ICMEs is decreasing with the solar distance, $D$, on average faster than in the $\mathrm{SW}\left(n_{\mathrm{SW}} \propto\right.$ $D^{-2}$ and figure 4 ). The density within 0.3 to $5 \mathrm{AU}$ decreases typically at between $D^{-2.3}$ and $D^{-2.6}$ [with Helios, WIND, ACE and Ulysses spacecraft, 1, 10, 24], with the exception of the recent results of [2] obtained only with Ulysses. Indeed, a blob of plasma in an ICME expands in all directions in contrast with a blob of plasma in the SW which expands mainly in the latitude and longitudinal directions (the SW has a nearly constant radial velocity for $D \geq 0.3 \mathrm{AU}$ ). The expansion in the radial direction (away from the Sun) is detected in situ by the temporal evolution of the radial velocity component (figure $5 b$ ). ICMEs are on average denser than the $\mathrm{SW}$ in the inner heliosphere, while because of their over expansion, they become less dense than the solar wind average in the outer heliosphere. However, this is only a weak effect and this statistical property cannot be used to identify ICMEs in the SW.

The ICME radial expansion velocity, $\Delta V_{x}$, is defined by the difference of velocity between the front and the back. $\Delta V_{x}$ is highly variable from one event to another one, even for MCs traveling in a "quiet" SW. It ranges from low values to a few $100 \mathrm{~km} / \mathrm{s}$ (figure 6a). However, a small fraction of MCs are in compression, while about half of MCs have strongly distorted velocity profile [far from linear with time, 26]. Typically in such cases, the $\mathrm{MC}$ is overtaken by a fast stream (or in few cases by another MC, section 5). Another characterization of the expansion (or compression) is achieved by defining a non-dimensional expansion factor $\zeta=\left(\Delta V_{x} / \Delta t\right) D V_{c}^{-2}$ ( $\Delta t$ is the MC duration, $D$ the heliocentric distance, and $V_{c}$ the velocity of the MC centre). In contrast to the broad distribution of $\Delta V_{x}$, the distribution of $\zeta$ is narrow for MCs that are not overtaken (figure $6 \mathrm{~b}$ ), showing that all these MCs have a typical non-dimensional expansion rate. Moreover $\zeta$ is independent of the magnetic field strength, of $D$ and of the size of the analyzed MCs $[22,26]$.

Why do MCs have a typical non-dimensional expansion rate? A theoretical analysis [23] shows that $\zeta$ is determined by the total pressure balance between the MC and the surrounding SW. Of course there is not an exact pressure equilibrium because of the magnetic tension, the evolution and the jump of pressure at the shock (if present). Still the pressure inside a MC can only be a few times larger than in the surrounding SW, while the SW pressure decreases by a factor $\approx 10^{-3}$ when $D$ is multiplied by a factor 10 (the pressure typically change as $D^{-n_{P}}$ with $n_{P} \approx 2.8$, figure $7 \mathrm{a}$ ). With magnetic flux conservation, this pressure balance gives a flux rope radius increasing as $\approx D^{0.7}$. A more detailed flux rope model confirms this, and shows that the expansion rate is almost 

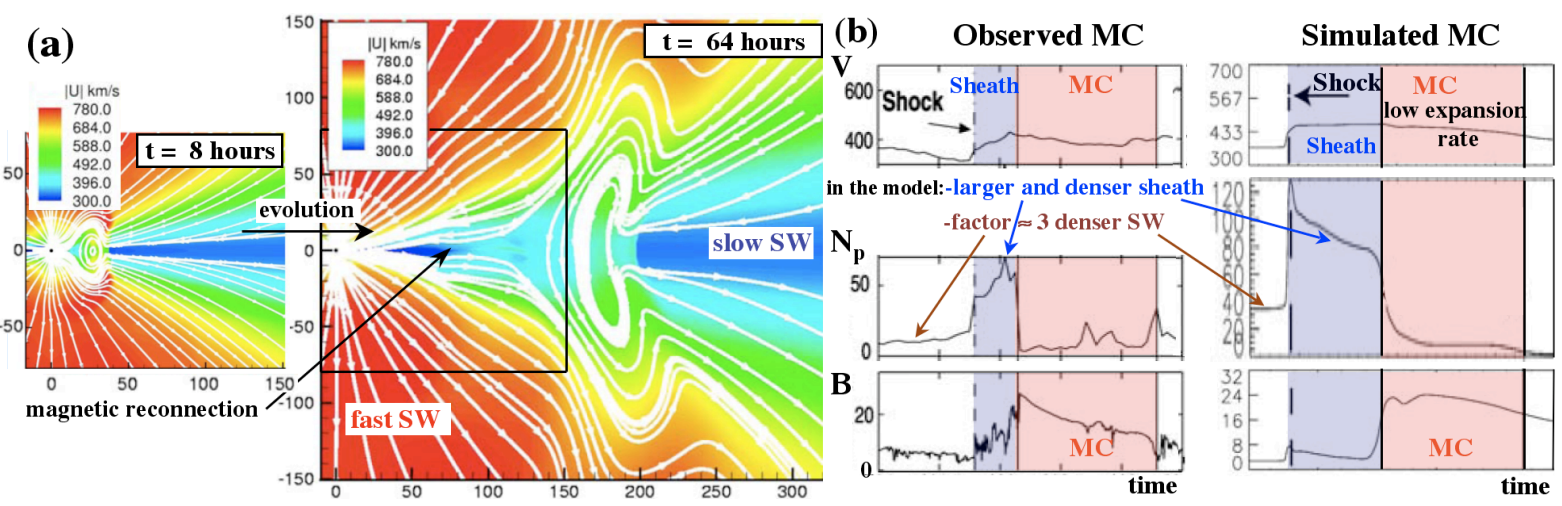

FIGURE 8. (a) 3D MHD simulation of a flux rope ejected from the Sun. The plots are in a meridional plane, orthogonally to the flux rope axis. Solid white lines are magnetic field lines computed in the 2D plane shown. The color shading indicates the plasma velocity. The flux rope cross section is strongly distorted by the latitudinal velocity gradient present between the slow and fast winds. (b) Comparison between a typical MC observation at $1 \mathrm{AU}$ and the MHD simulation. The simulation simulation has been rescaled to have a similar mean velocity, size (and hence duration) and maximum field strength as in the observed case [Adapted from 25].

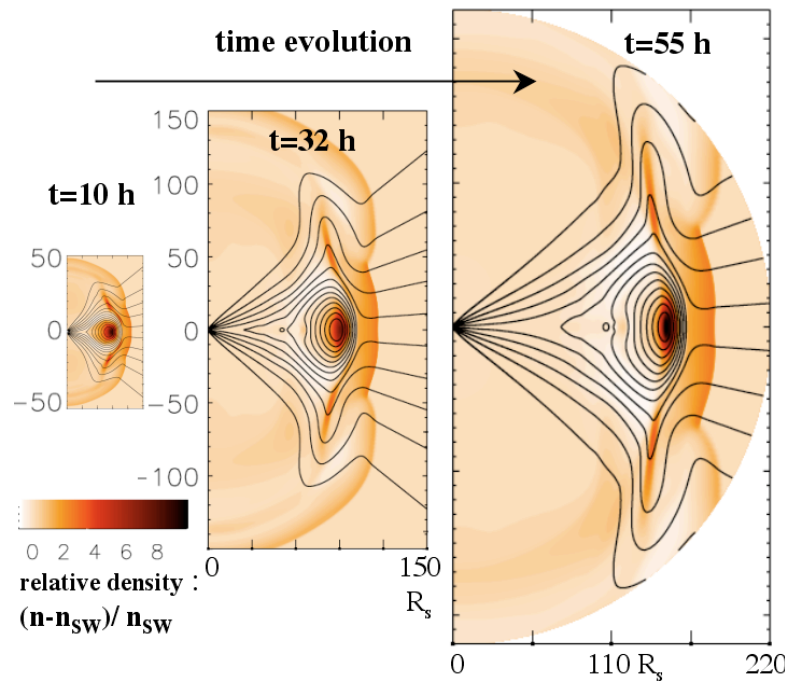

FIGURE 9. Axisymmetric 2.5D MHD simulation of a flux rope ejected from the Sun with a background SW as in figure 8 . Solid black lines are magnetic field lines. The color shading indicates the relative plasma density. Differences in the flux rope set up close to the Sun lead to a different evolution of the flux rope cross-section [Adapted from 7].

independent of the internal field model with conservation of magnetic flux or of magnetic helicity (so ideal or dissipative MHD). The velocity profile found in the model (figure $7 \mathrm{~b}$ ) is almost linear with time as in observations (figure 5b). From an ongoing study, the non-MC ICMEs have also the same expansion properties as MCs.

\section{DEFORMATION OF THE FLUX ROPE}

At the opposite of a ship, an ICME is a deformable structure. This is presently best studied using MHD numerical simulations with an unstable flux rope launched from the corona in a prescribed SW. In the simulations of $[6,21,25]$, the flux rope is large enough to propagate both in the slow and fast wind (figure 8a). The gradient of velocity induced a large deformation of the flux-rope cross section (set nearly circular close to the Sun).

The results of a numerical simulation can be compared to in situ observations by extracting the temporal evolution of the physical parameters at a fixed spatial location (figure $8 \mathrm{~b}$ ). The temporal profiles of velocity, density and field strength are comparable in the simulation and observations. However, the sheath region (accumulated SW plasma and B field in front of the flux rope) is much larger and denser in the simulation. Indeed the density of the SW was set 3 times larger than the typical value present in the slow SW. This is one origin of the strong distortion and low radial expansion of the simulated MC.

Other authors have found less deformed flux ropes (e.g compare the field lines shown in figures $8 \mathrm{a}, 9,11)$. The main differences with the simulation of figure 8 are a different initial flux rope, axisymmetric simulations, and, for figure 11, the absence of a latitudinal gradient of velocity in the SW. In figure 9, mostly the lateral borders of the flux rope and the magnetic field, encountered by the $\mathrm{SW}$, are severely distorted into two lateral extensions. If a spacecraft were to cross one of such extensions, it would detect some characteristics of an ICME (e.g. a rotating enhanced B field), but without the full characteristics of an ICME (e.g. the composition properties). This is also a case where the flux rope detection would be missed. We presently do not know which fraction of non-MC ICMEs 


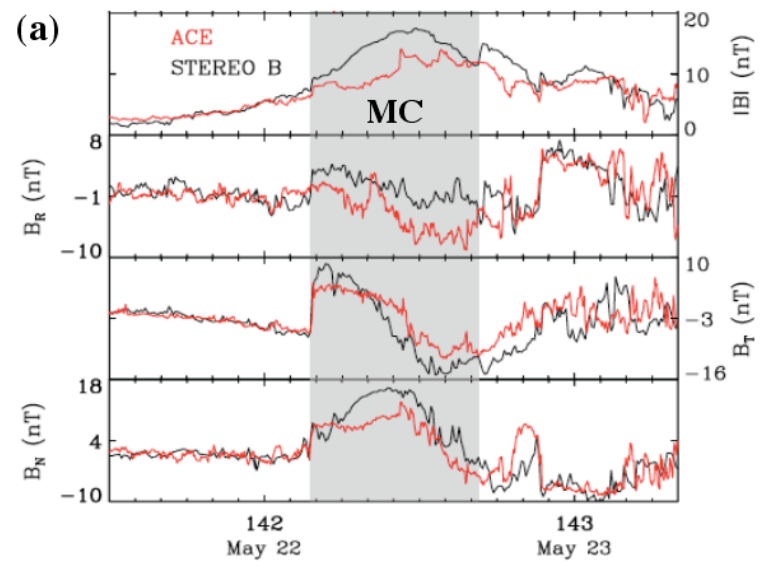

(b)

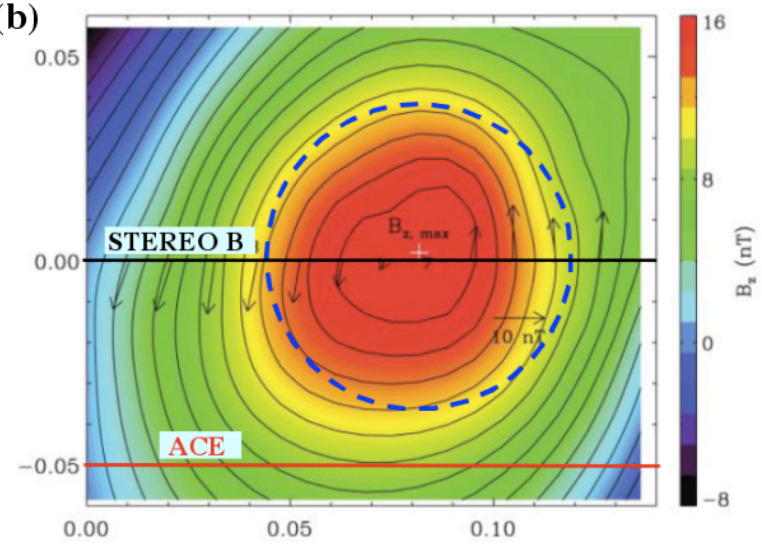

FIGURE 10. (a) Magnetic field strength and components observed by ACE and STEREO B across a MC. (b) Reconstructed cross section of the MC using STEREO B data and a 2.5D magnetostatic equilibrium within the MC moving frame. Black contours show the field lines projected orthogonally to the MC axis and the color shading indicates the value of the axial field. The trajectories of STEREO B and ACE, as well as a dashed circle, are superposed [Adapted from 27].

have a flux rope.

In situ observations indicate that some MCs can be flat [e.g. 28], but very flat configurations, such as in figure 8 , are rather exceptional based on current knowledge. Identification of such distorted flux ropes has so far not been successful [29]. Indeed, recent results of STEREO and ACE together with modelization $[27,30]$, rather indicates a relatively round cross section (at least in the central part, figure 10). This subject requires a deeper analysis of observations and the development of noncircular/elliptical models [31].

\section{OVERTAKEN ICMES}

During its outward travel, an ICME can be overtaken by a faster one $[33,34,35]$. This has been simulated (a)

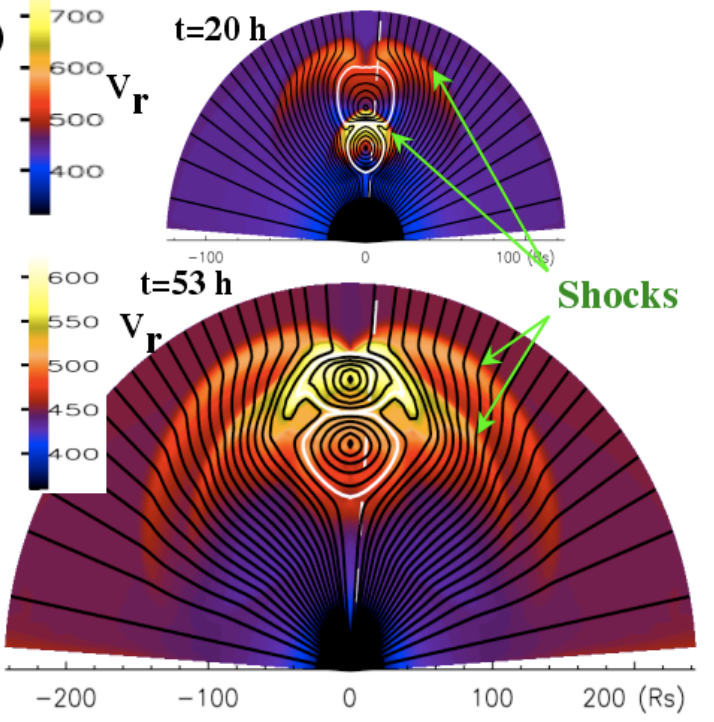

(b)

Results at $r=200$ Rs

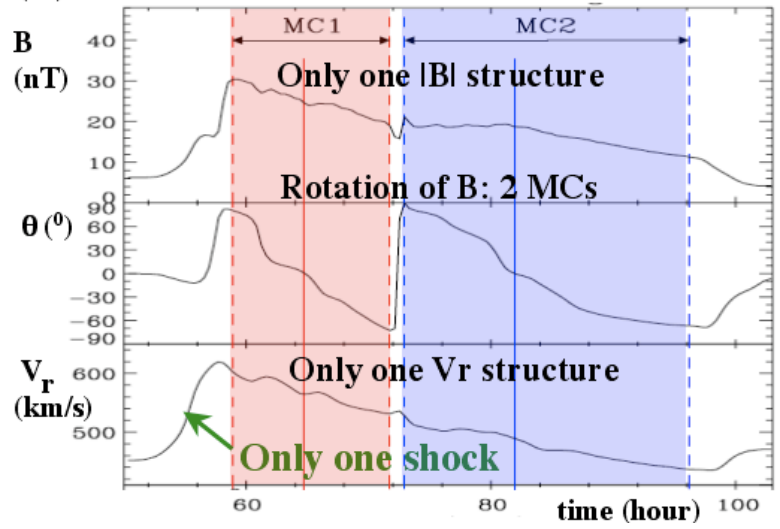

FIGURE 11. (a) Axisymmetric 2.5D MHD simulation of the interactions of two parallel flux ropes with the same characteristics. They are launched from the same part of the Sun with a time differrence $\approx 12 \mathrm{~h}$. The plots are in a meridional plane, solid black lines are magnetic field lines, and the color shading shows the radial velocity. (b) Time evolution of the magnetic field magnitude and latitude $(\theta)$, and of the radial velocity computed at a fixed spatial position (at this heliospheric distance the two shocks have merged). The two flux ropes are detected only on the orientation of the magnetic field $(\theta)$ as observed in the case shown in figure 12a [Adapted from 8].

by the launch of two successive flux ropes in numerical simulations similar to the ones described in the previous section $[8,36,37]$. A simple example with two identical flux ropes and with negligible reconnection is shown in figure 11a. The shock driven by the second flux rope catches up with, and rapidly propagates through the first flux rope (figure 11a) and finally merges with the shock driven by the first flux rope (figure 11b). The first flux rope is also compressed and flattened by the interaction with the second flux rope. After this interaction they 


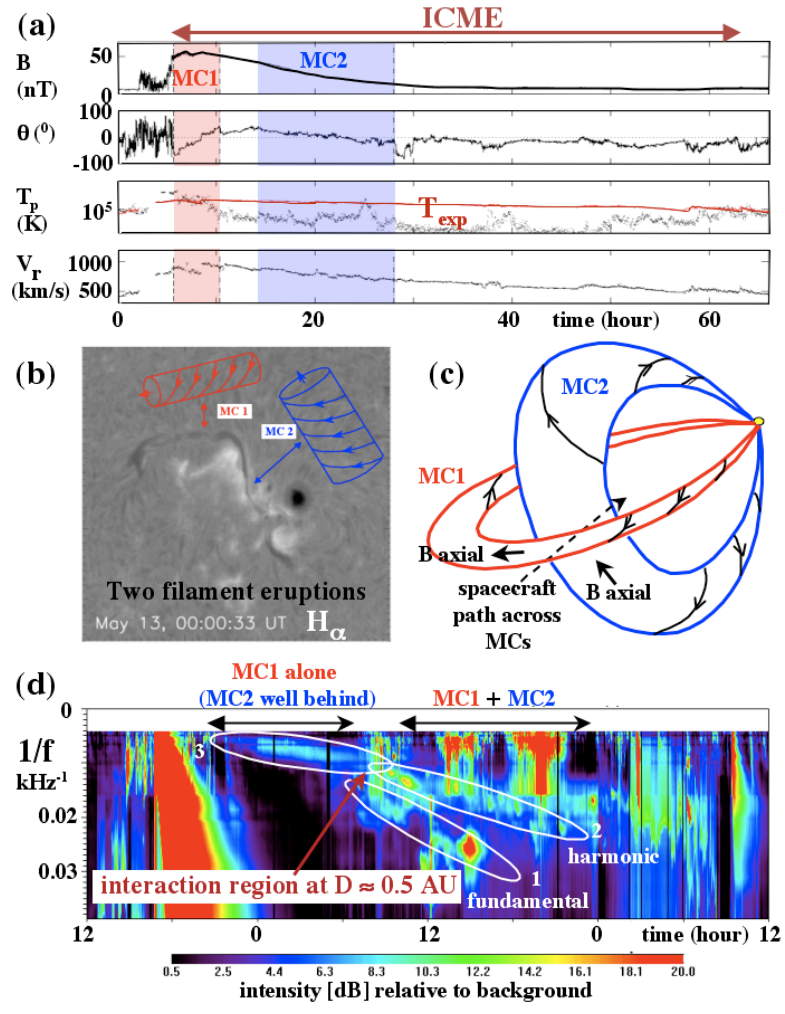

FIGURE 12. (a) In situ observations of an ICME with two interacting MCs inside (MC1 and MC2). (b) The eruption of two filaments are the solar sources of the MCs. The sketches of the flux ropes are added; their orientations, deduced from in situ observations, are comparable to the related filament orientations. (c) Sketch of the interacting flux ropes. (d) Dynamic spectral plot showing the radio emission of the interplanetary shocks (type II burst, encircled regions) in front of the first MC (MC1). During the first period of time (horizontal arrow), MC1 is traveling alone (MC2 is far behind), while after the interaction region they travel together, as observed in situ in panel (a). [Adapted from 32].

travel together as one entity.

Similar cases have been observed, but with flux ropes having different orientations and B flux [e.g. 32]. In the ICME shown in figure 12, two MCs have been identified, $\mathrm{MC} 1$ and $\mathrm{MC} 2$, in particular by the two different coherent regions of the magnetic field latitude $(\theta$ in figure 12a). The solar sources have been identified as two filament eruptions from the same active region (figure 12b). The shock in front of the first MC has been tracked through its radio emission (see beginning of section 2 and figure 12d). The shock has a significant change of velocity around $\approx 0.5 \mathrm{AU}$ (as deduced from the change of the frequency drift rate); this is interpreted as being due to the interaction between the two MCs (the faster and larger second MC pushing the first MC). This conclusion is in agreement with the time of the interac- tion estimated from the solar launch time and the in situ measured velocities of the MCs.

The overtaking structure can also be a fast SW stream. This is detected in situ by a fast velocity observed behind the MC, but also the fast stream can accelerate the trailing region of the MC [26]. As expected, most the overtaken MCs have a significantly slower expansion rate (lower $\zeta$ value) than those that are not overtaken shown in figure $6 \mathrm{~b}$. Nevertheless, surprisingly a few expand faster! The interpretation of this is the expected time dependence of the overtaking process: First, the MC is compressed. Second, the fast SW sweeps over the MC from the sides. Finally, the internal pressure of the $\mathrm{MC}$ is higher than the pressure in the surrounding SW (section 3) so it expands, tending towards the pressure and size it would have reached without interaction.

\section{EVOLUTION OF GLOBAL ICME QUANTITIES}

There are many interaction processes between ICMEs and the SW. Only four are reviewed above. These interactions modify the ICME mass and mean velocity, but also define the expansion rate and the shape of ICMEs. There is also magnetic reconnection between ICMEs and the overtaken SW field (in the sheath). This modifies two other global ICME quantities: its magnetic flux and helicity $[38,39,40]$. Moreover, interchange reconnection between SW and ICME magnetic field progressively removes the ICME connection with the Sun and plays an important role in reshaping the heliospheric field $[41,42]$. So the interaction with the SW indeed affects the main ICME physical properties. As a consequence, ICMEs progressively loose their identity as they move away from the Sun [e.g. 2].

\section{ACKNOWLEDGMENTS}

I thank the referee for his/her helpful comments on the manuscript.

\section{REFERENCES}

1. Y. Liu, J. D. Richardson, and J. W. Belcher, Planetary Spa. Sci. 53, 3-17 (2005).

2. R. W. Ebert, D. J. McComas, H. A. Elliott, R. J. Forsyth, and J. T. Gosling, J. Geophys. Res. 114, A01109 (2009).

3. R. F. Wimmer-Schweingruber, N. U. Crooker, A. Balogh, et al., Space Sci. Rev. 123, 177-216 (2006).

4. T. H. Zurbuchen, and I. G. Richardson, Space Sci. Rev. 123, 31-43 (2006).

5. L. Burlaga, E. Sittler, F. Mariani, and R. Schwenn, J. Geophys. Res. 86, 6673-6684 (1981). 
6. W. B. Manchester, T. I. Gombosi, I. Roussev, et al., J. Geophys. Res. 109, A01102 (2004).

7. E. Chané, B. Van der Holst, C. Jacobs, S. Poedts, and D. Kimpe, Astron. Astrophys. 447, 727-733 (2006).

8. M. Xiong, H. Zheng, S. T. Wu, Y. Wang, and S. Wang, $J$. Geophys. Res. 112, A011103 (2007).

9. G. M. Lindsay, J. G. Luhmann, C. T. Russell, and J. T. Gosling, J. Geophys. Res. 104, 12515-12524 (1999).

10. C. Wang, D. Du, and J. D. Richardson, J. Geophys. Res. 110, A10107 (2005).

11. D. B. Berdichevsky, C. J. Farrugia, B. J. Thompson, et al., Annales Geophysicae 20, 891-916 (2002).

12. M. J. Reiner, M. L. Kaiser, and J.-L. Bougeret, Astrophys. J. 663, 1369-1385 (2007).

13. S. Hoang, C. Lacombe, R. J. MacDowall, and G. Thejappa, J. Geophys. Res. 112, A09102 (2007).

14. A. P. Rouillard, this issue (2009).

15. N. Gopalswamy, A. Lara, S. Yashiro, et al., J. Geophys. Res. 106, 29207-29218 (2001).

16. J. Zhang, K. P. Dere, R. A. Howard, and V. Bothmer, Astrophys. J. 582, 520-533 (2003).

17. R. Schwenn, A. Dal Lago, E. Huttunen, and W. D. Gonzalez, Annales Geophysicae 23, 1033-1059 (2005).

18. R. Woo, J. Geophys. Res. 93, 3919-3926 (1988).

19. B. Vršnak, Annales Geophysicae 26, 3089-3101 (2008).

20. A. Borgazzi, A. Lara, E. Echer, and M. V. Alves, Astron. Astrophys. 498, 885-889 (2009).

21. N. Lugaz, W. B. Manchester, IV, and T. I. Gombosi, Astrophys. J. 627, 1019-1030 (2005).

22. P. Démoulin, M. S. Nakwacki, S. Dasso, and C. H. Mandrini, Solar Phys. 250, 347-374 (2008).

23. P. Démoulin, and S. Dasso, Astron. Astrophys. 498, 551-566 (2009).

24. M. Leitner, C. J. Farrugia, C. Möstl, et al., J. Geophys. Res. 112, A06113 (2007).

25. W. B. I. Manchester, T. I. Gombosi, I. Roussev, et al., J. Geophys. Res. 109, A02107 (2004).

26. A. M. Gulisano, P. Démoulin, S. Dasso, M. E. Ruiz, and E. Marsch, this issue (2009).

27. Y. Liu, J. G. Luhmann, K. E. J. Huttunen, and et al, Astrophys. J. 677, L133-L136 (2008).

28. M. Vandas, E. P. Romashets, and S. Watari, "Connecting Sun and Heliosphere," in B. Fleck, T.H. Zurbuchen, $H$. Lacoste (eds.), Solar Wind 11 / SOHO 16, ESA SP-592, 159.1 (on CDROM), 2005.

29. M. J. Owens, J. Geophys. Res. 111, A12109 (2006).

30. C. Möstl, C. J. Farrugia, H. K. Biernat, et al., Solar Phys. 256, 427-441 (2009).

31. P. Démoulin, and S. Dasso, Astron. Astrophys. in press, DOI: 10.1051/0004-6361/200912645 (2009).

32. S. Dasso, C. H. Mandrini, B. Schmieder, et al., J. Geophys. Res. 114, A02109 (2009).

33. L. F. Burlaga, R. M. Skoug, C. W. Smith, et al., J. Geophys. Res. 106, 20,957-20,977 (2001).

34. Y. M. Wang, S. Wang, and P. Z. Ye, Solar Phys. 211, 333-344 (2002).

35. C. Farrugia, and D. Berdichevsky, Annales Geophysicae 22, 3679-3698 (2004).

36. N. Lugaz, W. B. Manchester, IV, and T. I. Gombosi, Astrophys. J. 634, 651-662 (2005).

37. N. Lugaz, J. Atmos. Sol. Terr. Phys. 70, 598-604 (2008).

38. S. Dasso, C. H. Mandrini, P. Démoulin, and M. L. Luoni, Astron. Astrophys. 455, 349-359 (2006).
39. S. Dasso, M. S. Nakwacki, P. Démoulin, and C. H. Mandrini, Solar Phys. 244, 115-137 (2007).

40. P. Démoulin, Annales Geophysicae 26, 3113-3125 (2008).

41. N. U. Crooker, R. Forsyth, A. Rees, J. T. Gosling, and S. W. Kahler, J. Geophys. Res. 109, A06110 (2004).

42. N. U. Crooker, and T. S. Horbury, Space Sci. Rev. 123, 93-109 (2006). 